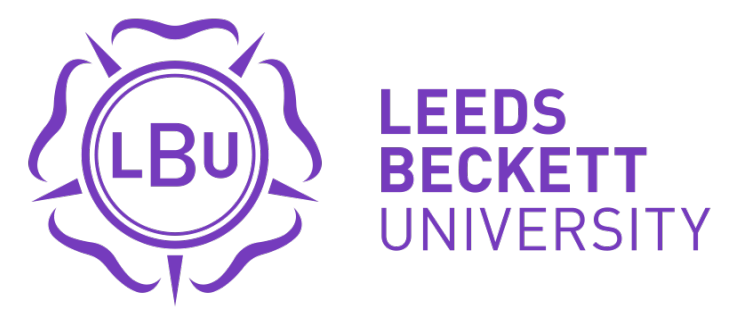

Citation:

Ibrahim, R and Towndrow, H and Monekosso, D (2020) Three loT Wearables in Six European Cities! Reality and Perception. In: Internet of Things, Infrastructures and Mobile Applications. IMCL 2019. Advances in Intelligent Systems and Computing, 1192 . Springer International Publishing, pp. 425-436. ISBN 9783030499310 DOI: https://doi.org/10.1007/978-3-030-49932-7_41

Link to Leeds Beckett Repository record:

https://eprints.leedsbeckett.ac.uk/id/eprint/7268/

Document Version:

Book Section (Accepted Version)

This is a post-peer-review, pre-copyedit version of an article published in Internet of Things, Infrastructures and Mobile Applications. IMCL 2019. The final authenticated version is available online at: http://dx.doi.org/10.1007/978-3-030-49932-7_41

The aim of the Leeds Beckett Repository is to provide open access to our research, as required by funder policies and permitted by publishers and copyright law.

The Leeds Beckett repository holds a wide range of publications, each of which has been checked for copyright and the relevant embargo period has been applied by the Research Services team.

We operate on a standard take-down policy. If you are the author or publisher of an output and you would like it removed from the repository, please contact us and we will investigate on a case-by-case basis.

Each thesis in the repository has been cleared where necessary by the author for third party copyright. If you would like a thesis to be removed from the repository or believe there is an issue with copyright, please contact us on openaccess@leedsbeckett.ac.uk and we will investigate on a case-by-case basis. 


\title{
Three IoT Wearables in Six European Cities! Reality and Perception
}

\author{
Rasha Ibrahim \\ Leeds Beckett University, Leeds, UK \\ rasha.ibrahimaleeedsbeckett.ac.uk \\ Holly Towndrow \\ Leeds Beckett University, Leeds, UK \\ H. Towndrow@leedsbeckett.ac.uk \\ Dorothy Monekosso \\ Leeds Beckett University, Leeds, UK \\ D.N.Monekosso@leeedsbeckett.ac.uk
}

\begin{abstract}
In the last decade, the Internet of Things (IoT) technology has attracted lots of attention. This paper evaluates the impact of three different IoT technologies represented in three types of wearables; Smart Glasses, Tracking Devices, and Crowd and Staff Wristbands. The deployment of these devices took place in a number of cultural and sports events in six European counties, as part of the European Commission funded project; MONICA. The analysis focused on the usability of the wearables and their impact on safety and security.
\end{abstract}

Keywords-IoT, Wearables, Mobiles, Usability, User-Experience, Safety,

Security, Privacy, HCI 


\section{Introduction \& Scope}

The Internet of Things (IoT) is regarded as one of the prominent technologies of this century which has attracted lots of attention in society, industry and academia. Examples of IoT devices include IoT enabled sensors with different data capabilities (video, audio and other data types), resource constraints (wearables, Smartphones and Smartwatches), bandwidth (UWB and M2M), costs and deployment (wearable, mobile, fixed, and airborne) in addition to actuators (lights, LED, cameras, alarms, drones, and loudspeakers). Wearable devices with sensing, actuating, localisation, and communication capabilities can support several applications by being deployed as actuators (LEDs) through automated closed-loop solutions and can be integrated with more powerful Smartphones and/or Smartwatch apps.

IoT can be used in a wide range of industries. In this paper we are interested in IoT technologies used for smart living, in particular, tourism industry for the purpose of safety and security in large festivals and sports events. This paper investigates the deployment of such technologies in 6 European cities; Leeds (UK), Copenhagen (Denmark), Hamburg (Germany), Turin (Italy) Lyon (France), and Bonn (Germany). This deployment took place as part of the European Commission funded project; MONICA (Management of Networked IoT Wearables- Very Large Scale demonstration of Cultural Societal Applications). The project is funded through HORIZON 2020 Framework Program for Research and Innovation; grant agreement No 732350 .

In the MONICA project, IoT technologies are used for a number of applications including sound monitoring and control, crowd safety and security, and citizen management and innovation. This is performed by exchanging information via IoTenabled devices deployed at cultural events. These can be worn (smart wristbands, glasses), mobile (smartphones, airships) or fixed (video cameras). IoT-enabled features mean that such devices have sensors that can wirelessly connect to the internet, communicate and instigate actions. This paper will focus on three IoT technologies; smart glasses, smart wristbands, and tracking technologies. The evaluation aspects covered include; usability of such devices, fit for purpose, and users' perception regarding privacy and data protection aspects accompanied by such technologies.

In the following sections, we will briefly describe the events taking place in the six European cities where the MONICA IoT technologies are being deployed. We will refer to these events as 'pilots'. Then we will briefly describe the three mobile technologies assessed, and explain the aspects covered in the assessment. Afterwards, the actual assessment is reported followed by our conclusion and lessons learned.

\section{Pilots Context}

This section provides a brief description of the events in the six European cities where the IoT wearable technologies are deployed. 


\subsection{Leeds Rugby Varsity Game}

Leeds Varsity is an inter-university sports competition between arch rivals University of Leeds and Leeds Beckett University. It begins in January with events throughout the year and finishes with one final day in October hosting over 60 fixtures across 25 different sports. The event culminates with the Men's Rugby Union Grand Finale at Headingley Carnegie Stadium in the evening, which attracts an audience of over 15500 students each year (LeedsVarsity, 2019).

\subsection{Movida}

The nightlife in Turin, which is known as "Movida" happens every day, particularly at the weekend, where large crowds group together in the San Salvario District to have fun and socialise. This movement began in the 1990's and has grown ever since. As a consequence of Movida, the 7300 residents living in the area are being affected by the disturbance and noise from the crowd (MONICA-Project, 2019). To reduce these consequences, Turin have produced restrictions such as opening hours, the sale of takeaway and alcoholic drinks, consumption of alcoholic drinks in glass/cans around a public area. The main goal for MONICA at Movida is to correlate sound with crowd data and handle security incidents, supporting the municipality to find an adequate balance between safety and security, amusement and quality of public spaces.

\subsection{Winter DOM}

The Hamburger DOM is Northern Germany's biggest funfair with 7-10 million annual visitors during the 91 DOM days (MONICA-Project, 2019). The funfair takes place in the premises of the Heiligengeistfeld with a total of around 251 attractions and 60 food stalls. The area is $160.000 \mathrm{~m}^{2}$, fenced, with nine free entrances. In the past three Winter editions, there have been an average of 2-3 million visitors. For Winter DOM, MONICA was used for Crowd and Capacity Monitoring, locating staff, and managing safety incidents.

\subsection{Fête des Lumières (Festival of Lights)}

Fête des Lumières is a free cultural event hosted by the city of Lyon. It is a festival composed of light installations with, for some of them, sound animations. The installations are located in well-known public areas of Lyon (places, streets, squares, parks). Around 50 light performances take place sometimes with sound playing. Depending on the year, the event can last 1 to 4 days and people can use free public transportation to admire the shows. The timeslots are usually from 6p.m. to midnight. This site welcomed approximately 2000000 visitors in 2016, this low attendance was caused by terror risk (Jahn, et.al, 2018). The 2015 edition was cancelled for this reason too. In the previous editions 3-4 million visitors were present during the days of the 
event. MONICA was used for sound monitoring, crowd and capacity monitoring, locating staff, and managing security incidents.

\subsection{Friday Rock Tivoli Gardens}

Friday Rock (Tivoli) is Copenhagen's famous amusement park located in the heart of the city. The park opened in 1843 and is the second-oldest amusement park in the world. The park offers a wide variety of rides, shows and attractions with around 4.5 million visitors annually. From April through September, Tivoli organises Friday Rock, a recurrent annual open-air concert-series with national and international performers. The capacity of the whole concert area is 25.000 people and the main stage area for Friday Rock is around $2.700 \mathrm{~m} 2$. MONICA was deployed in Tivoli for sound monitoring and control, crowd and capacity monitoring, missing person, and managing security incidents.

\subsection{Pützchens Markt}

Pützchens Markt (PM) is a street festival that takes place in Bonn every year in the second week of September. For five days, the event offers all kinds of attractions such as traditional merry-go-rounds, more than 550 commercial stalls and two stages. This massive offer extends over the length of $4,5 \mathrm{~km}$ in an area of $80.000 \mathrm{~m} 2$. Pützchens Markt attracts around 1,35 Million visitors (Jahn et al., 2018). MONICA was deployed in PM for crowd and capacity monitoring, and for locating staff.

\section{IoT Wearable Technologies}

\subsection{Smart Glasses}

The smart glasses deployed in the MONICA project were produced by Optinvent. They are ORA-2 products which allow a full see-through feature. The ORA-2 product feature includes:

- $\quad$ Standalone wireless product with embedded battery, weight $\sim 90 \mathrm{~g}$

- Runs generic Android Kitkat (4.4.2) and has open platform to create and execute any Android applications.

- $\quad$ Use Arm 9 Dual Core 1.2GHz processor with GPU \& 5.3 Gb Storage Memory and 1Gb Flash Memory D3.1 IoT Enabled Devices and Wearables 1 Document version: 1.0 Page 39 of 60 Submission date: 2017-12-27 
- Track pad on rigid side of the frame (True Mouse not only swipe)

- Embedded $1200 \mathrm{mAh}$ rechargeable battery

- Can be worn over most of the user's glasses.

The glasses have the following sensors

- 5 megapixel Auto-Focus camera

- Light sensor to adjust automatically display brightness to environment brightness

- Active GPS

- 9 axis sensors (Gyro/Accelerometer/Compass; MPU9250 from Invensens)

- Low noise microphone and mono audio out through $\mu$ USB provided accessory

$>$ Connectivity:

- $\quad$ WiFi b,g,n; $2.4 \mathrm{GHz}$

- BT 4.0 Low Energy

- $\mu$ USBS 2.0 for charging, data exchange and transporting mono audio to an audio headset accessory

Display:

- See-through Display Feature with 50\% photopic transparency

- $800 \times 480$ pixels native resolution RGB colour display with 42 pixel/deg

- Field Of View of 22deg with Flip-Vu feature to move the image location 5 deg up, centred to eye sight and 20deg down

- High brightness (Schoneveld et al., 2017).

\subsection{Smart Wristbands}

The wristbands used in MONICA are produced by DEXELS. They monitor both crowd and staff.

\section{A. Crowd}

In order to deploy, the Crowd Wristband requires -

- Base stations

- Ethernet (PoE) network between base stations

- Sufficient spots to mount base stations, which are at least $4 \mathrm{~m}$ high

- Professional installation

- Production office space

The features of the Crowd Wristband include brand activations, access control and cashless payments, which is captured using RFID. Other features include profile exchange, matching, friend finder, child finder, voting and alerts. Crowd monitoring also takes place, so there is a display of heat maps to show busy areas, and it also helps 
with the visitors' journey. LED is another feature of the Crowd Wristband. This allows a group notification or an individual notification, and also enables for a light show.

\section{B. Staff}

There are several features regarding the Staff Wristband. One feature is the localisation of staff, which adopts a very high accuracy. Staff are also able to send short messages amongst each other, which the recipient can acknowledge they have received the message. The Wristband also includes a buzzer and sound.

In order to deploy, the Staff Wristband requires -

- Anchors

- Ethernet (PoE) network for at least 1 anchor

- Sufficient spots to mount anchors are least $6 \mathrm{~m}$ high

- Professional installation

\subsection{Tracking Devices}

The tracking device assessed in this paper is LoRa Tracking device. The functionality of this device is to track individuals such as event staff. It has features such as communication buttons, which enables the user to send and receive a text.

\section{Assessment Aspects}

This section discusses the aspects according to which the evaluation of the IoT wearable technologies took place.

\subsection{Wearables Usability}

A wearable is a technology which is worn on the body, and is one of the devices at the forefront of new technologies. The technology for wearable gadgets has vastly grown over the years, particularly in healthcare. Smart watches, for example, are one of the major wearables that can be used today.

Razvan (2015) stated in "Designing a User Experience for Wearable Devices", that the early process of wearables included two types - eyewear (smart glasses) and smart watches. Key features of eyewear is the ability to navigate, track location and provide route information, take pictures and record videos, as well as performing online searches. Smart watches are more focused on tracking physical actions, such as running, walking etc. Most smart watches now provide navigation, music listening abilities, the ability to make payments and calls and send texts. 
Comparing the two, smart watches have a higher amount of apps that are available, compared to smart glasses, and they're often cheaper (Razvan, 2015).

Razvan (2015) also suggests that to make the device useable, you must keep the device small, easy to attach and comfortable. Sticking to the core features also makes the devices usable, not overcomplicating things and minimal elements on the screen enables the user to interact easily.

It's important that the wearables are minimal in weight and are comfortable. In a report by Knecht, et al (2016), they also elude to the idea that wearables should be comfortable and lightweight. The study undertaken showed that participants were affected by different types of material and this had an implication on whether they would use the devices in the future. For example, parts of the wearable were described as an obstacle and burden.

On the other hand, a small device isn't able to produce a long battery life and distinctive apps. Liang et al. (2018) produced a study on system usability scale evaluation of mainstream wearable fitness devices. The study evaluated that the usability of mainstream wearables has a negative perception from users and their need to buy wearables is low. In addition, Liang et al. (2018) suggests that manufacturers of these wearable devices should further their effort to develop innovative and unique functions, and improve the overall usability by adding cognitive behaviour change techniques into the system.

\subsection{Fitting for Purpose}

The IoT mobile devices reported in this paper were mainly used to improve conditions in large-scale events attended by thousands, and in some cases millions of people. Accordingly, the wearables were assessed according to their fit for purpose. This was done in relation to the project use-cases in terms of crowd monitoring, sound monitoring, managing security incidents, and health incidents, and locating staff.

\subsection{Privacy and Data Protection issues}

An important aspect that was evaluated in some of the wearable devices reported here are the privacy and data protection aspect. Some of the wearables collect location data or other personal information of either the staff or audience using them. We needed 
to assess how users feel about capturing their personal information for the purpose of improving the service provided by the event organisers in such large-scale cultural events.

\section{$5 \quad$ IoT Technologies Evaluations}

This section reports the assessment of three IoT wearables; Smart glasses, smart wristbands, and tracking devices in six European cities deploying IoT technologies for the MONICA project.

\subsection{Smart Glasses}

Thirteen users participated in the deployment of the smart glasses in Rugby Varsity Game in Leeds (UK), Tivoli in in Copenhagen (Denmark), Movida festival in Turin (Italy), and Festival of Lights Lyon (France).

In Leeds, OPTINVENT smart glasses were used by a professional steward with an extensive experience as a security staff. To evaluate the smart glasses, we conducted a semi-structured interview with the steward. This method allowed us to have a one-oone conversation with the steward and get his thorough feedback about the smart glasses and its effectiveness as a mobile technology to ensure safety and security in the stadium.

Normally, the glasses have a joystick that the user can use. However, we decided that the steward use the glasses touch pad on the frame instead so his hands were free. The steward felt the smart glasses were easy to use and comfortable. He felt they helped him to quickly and effectively complete his work. The clarity of images of the IoT smart glasses were much better than the use of normal body-cam in terms of targeting and focus. The steward felt the glasses could definitely help to improve the security operations in the stadium especially in relation to:

- Bags search: The glasses gave a closer and a more accurate view, better than just the eyes. "I felt by just looking through the bags via the smart glasses, as if my hands were already inside the bags".

- Security and health incidents: Specially using the video mode, the smart glasses helped the steward to feel both match spectators and the objects much closer. Accordingly, the abnormalities were easier to detect.

The steward commented that the memory card finished after 4 hours because he used the video mode a lot. All the images and videos captured by the glasses were remotely stored at a laptop in the MONICA control room where Leeds Beckett University staff were located. The steward suggested some adjustments to the glasses bridge over the nose for more comfortability. He recommended to integrate the headset with the glasses so that the stewards won't have two pieces on the head to manage. 
Regarding stability of connectivity, because of some construction work at the Stadium on the day, the internet connection wasn't working everywhere in the stadium, so the steward couldn't send pictures and videos when he was far from activated access points. But the glasses worked well for him everywhere. Near the access points where the internet connection was working well, the steward was able to send all videos and photos taken to the control room remotely and instantly.

In Turin, at the nightlife of San Salvario District, Movida, three smart glasses were used by Turin local police officers. A questionnaire was distributed among them to get their evaluation of the smart glasses, and if they believe the glasses can be an effective tool for local police patrolling during Movida.

The MONICA server sent a message to the police officers regarding a suspicious individual. The officers checked an Identity Document (ID) of the suspect by exchanging photos, both from the officers to the MonicOra server and vice versa. The ID was then verified by the station and then the intervention was closed. The first attempt resulted in the officers receiving the messages from the MonicOra server, however the MonicOra server did not receive the messages from the officers. This is down to a connectivity issue between the server and the glasses. During the second attempt, all messages between the glasses and MonicOra server and vice versa were exchanged and received.

Nine participants in total filled the questionnaire which was focused on the usability of the smart glasses and how they aided the end users during their work. This questionnaire helped evaluate the impact that the smart glasses have on both the end user and the work environment. About the end users were made up of both male and females, from different professional backgrounds within the police sector.

The table below summaries the characteristics of the participants.

\begin{tabular}{|l|l|l|}
\hline $\begin{array}{l}\text { Total } \\
\text { Participants }\end{array}$ & Gender & Job Position \\
\hline 9 & Male (7) Female (2) & Police officer (3) \\
& & Police officer and analyst (1) \\
& & Superior police officer (1) \\
& & N/A (4) \\
\hline
\end{tabular}

\section{Smart Glasses Efficiency:}

The objective of the smart glasses is to improve the efficiency of the user's work in terms of securing the event. $56 \%$ of participants either agreed/strongly agreed that using the smart glasses ensured them that they could effectively complete their work. Three participants disagreed with this. Regarding the easiness via which the participants learnt how to use the smart glasses, $78 \%$ of all participants agreed that it was easy to learn how to use the smart glasses, and only one participant found difficult to understand how it worked. In terms of the usability of the glasses, 56\% agreed that they were satisfied with how easy it was to use the smart glasses, only one participant 
disagreed. 33\% of participants were neutral on this. Regarding the speed of performing tasks, participants were also asked whether they could complete tasks quickly using the smart glasses. Three out of nine participants believed they could complete tasks quickly, whilst another three participants were neutral. The remaining three participants believed that it took them extra time to complete their tasks using the smart glasses. When the smart glasses comfortability was investigated, $67 \%$ of all participants felt they were comfortable with using the smart glasses. Only two participants felt they weren't comfortable using them. One justified that by the weight of the glasses, and the too high overall dimensions. The final participant was neutral. When making a mistake, $2 / 3$ of the participants felt by using the smart glasses they could recover easily and quickly. One participant disagreed with this statement and two stayed neutral.

\section{Distinct Features:}

The participants were asked to determine their most important feature of the smart glasses. Only five participants answered this question, and they praised the innovation. With quotes such as "undoubtedly innovative" and with some commenting on the freedom of hands they experienced wearing the smart glasses and how helpful it was to have their hands almost completely free. The most appreciated feature of the smart glasses was the ability to share data simultaneously with colleagues remotely. Participants thought that the idea of sharing real-time images, as well as also recording, was an important feature of the smart glasses. So, they stressed on the importance of both the video and the camera. Regarding safety and security incidents, $67 \%$ of the participants confirmed that the smart glasses were useful for detecting such incidents. Regarding reporting, $44 \%$ of the participants believed that the glasses would help report an incident. The smart glasses were regarded as a "new communication tool".

\section{Additional Functions:}

The participants were also asked what capabilities they would have expected the smart glasses to have. Out of the six participants who answered this question, one participant stated that the smart glasses in their current status covered all functionalities they needed and that they "would not expect to see anything else". One participant suggested to add an emergency button, and another participant would have liked to see voice commands as an option. Four participants stated that they would have liked to be able to film in real-time. It's worth mentioning that such functionality is already included in the smart glasses, and was deployed in other pilots (such as Leeds), but was not used in Movida due to unstable connection the deployment suffered in November 2018. Connectivity was an issue with two participants. One participant stated that there was a limited possibility of writing messages without a remote controller. Whilst another stated the modem/joystick and the smart glasses were not something they liked.

Regarding the physicality's of the glasses, two participants stated the weight was an issue, as well as too high overall dimensions. Overall, out of nine participants, five were satisfied with the MONICA smart glasses.

In Lyon, at the festival of lights (Fete des Lumieres), three smart glasses were deployed and a questionnaire was conducted to evaluate the participant's opinions on this technology. For the impact analysis, several aspects have been considered:

\section{1) Smart Glasses aiding participants:}


The participants were asked what was the most important thing they felt the smart glasses helped them in. One participant liked the information collection in real time, as well as the ease of transmission; another participant found that the keypad was nice to use, though takes up use of one hand; finally, the last participant appreciated the fast GPS tracked messages.

2) Additional Functionalities:

The participants were asked, in addition to the functions the smart glasses currently provide, what else they expected the glasses to feature. The following feedback was received: • Further upgrades of the product, such as "send pictures to nearby glasses" and direct contact between users (skipping the COP) - Improve physically: the glasses are rigid and cannot be folded or stored easily when not being used. $\bullet$ Inboard display of the COP, a map which displays the users position.

\section{3) Cons of Smart Glasses:}

To gain further feedback, the participants were also asked to mention two things they did not like about the MONICA smart glasses. The following feedback was received:

- Important to be able to focus on a single point because it was hard to balance viewing with environment.

- The side view is limited by the battery icon.

- Storing was an issue.

- Smart glasses are only for people with right eye as the main eye. Those with left guiding eye couldn't use.

\section{4) General feedback:}

Out of three participants, only one participant gave general feedback. The general comments were that the smart glasses are weather dependent; when raining or snowing, they can't be used because they aren't 100\% waterproof and feature no infra-red camera. Furthermore, the smart glasses are unable to be stored in a pocket or fit around the end user's neck. The user also referred to the messages received, stating that there were too many to scroll. Finally, the WIFI limited the covered area - so for festivals with more than one venue, this can be an issue.

\subsection{Smart Wristbands}

Six crowd wristbands were deployed in Tivoli Gardens in Copenhagen for the purpose of locating a missing person. A questionnaire has been distributed to the endusers for evaluating the wristbands. Regarding the usability of the wristbands, $100 \%$ of the participants were happy with the wristband weight. Also, $67 \%$ of the participants agreed on the wristbands suitability for a child in terms of their weights but two participants were neutral. All the participants except one (who was neutral) agreed that it was very easy to operate the wristband. Regarding the comfortability of the wristbands, $100 \%$ of the participants felt they were comfortable to wear. $50 \%$ of the 
participants felt the wristband helped improve the communication with their colleagues. The other half were neutral. In regard to the issue of privacy and data protection, the participants stated that they do not mind being tracked as they understood that the purpose of the tracking is for their own interest, as shown from some of their quotes below:

"It's totally fine, it's for my safety".

"I'm okay with it".

\subsection{Tracking Devices}

Fifteen LoRa tracking device were deployed in Pützchens Markt in Bonn (Germany) for the purpose of monitoring the location of staff. Users were asked to evaluate the feasibility of the LoRa Tracking devices based on stability, accuracy, uptime, reliability and range. Feedback included improvements for the app. For example, it was suggested that it would be nice to have the map on one screen and the information on a second screen. It was also suggested that "early warnings" before an area gets too full would be a good feature to add. The tracking marker should also be blurred or indicate to show when a device didn't transmit for a prolonged period of time. Furthermore, the locations often jumped, which provided inaccurate results. The tracking device as a whole could have improvements. For instance, the device should be more robust and smaller in size. As well as this, the on/off button is easily switched which could hinder the user. Extensive communication options would also be a preferred feature to add to the device. The ability to send an emergency signal by one push is highly desired. Finally, the user would require faster updates and an extensive battery life.

In Winter-DOM in the city of Hamburg (Germany), the initial plan was to deploy several trackers for this pilot demonstration, for use by police, medics and security staff. Privacy and security concerns meant this was not possible at that time as this needs better preliminary planning ahead of the following DOM in 2019 to allow for larger deployment of devices. Accordingly, only six trackers were deployed in 2018. They were successfully tested on the first pilot at the DOM. For the evaluation of the tracking devices, several aspects have been considered:

1) Privacy: in terms of privacy, the participants were asked about the way they felt about other staff/people tracking their location. 100\% of participants who answered the question believed that for work purposes they had no concerns and would continue to wear the device. Whilst the device in the participant's opinions did not invade privacy in the work place, $2 / 3$ of the participants that answered believed that it would be more concerning should the user not be able to switch the device off during home time.

2) Usability and ease of use: $75 \%$ of the participants believed that the device was easy to use, with one participant being neutral on this question. $50 \%$ believed that the device was of sufficient weight. This is further echoed by one participant suggesting she forgot she was wearing the device during their day. 
$75 \%$ of the participants was neutral when commenting whether the tracking device was comfortable to wear. One participant disagreed and believed that the tracking device was "too big" and had "no possibilities to attach" to the user. Whilst $75 \%$ of participants were neutral regarding the comfort of the tracking device, all the participants agreed that the device did not hinder or obstruct them from doing their normal daily job. The participant who found the device "too big" also agreed the device did not obstruct or hinder her duties.

3) Overall performance: when asked if the tracking device met their expectations, $75 \%$ of participants stated that their expectations were met with some suggesting some improvements as reported in the section below. Overall, the participants believed the device worked as expected and for most parts did what was anticipated. Only one participant stated the device did not do as expected.

4) Improvements: In regards to battery life, whilst 50\% of participants clarified that the device had a substantial battery life, $100 \%$ of participants agreed that showing the battery life/status of the device would have been incredibly important. A visual battery percentage was the participant's suggestions. Device on/off: $75 \%$ of participants stated that not being able to switch on/off the device was an issue. To further improve the device, it has been suggested to add an on/off button, enabling the user to manage the devices status. They would like to be able to switch the device off even for a small period of time. $50 \%$ of participants stated that the on/off switch would be useful when off duty.

\section{Summary}

The paper presents assessment of the IoT wearables used for smart living in the tourism industry, in specific, in festivals and sports events. The technologies assessed were smart glasses, wristbands, and tracking devices. The technologies were evaluated in terms of their usability, and impact on safety and security. The results showed that in general, most of the users of the wearables found them easy to wear and operate with the exception of situations when there were issues with connectivity. In regards to the users of the wearables, all the devices were assessed by the real users with the exception of child visitors wristbands, which were evaluated by adults. We believe, it is essential that children of different age groups conduct the assessment of the devices putting into consideration all ethical consideration necessary. In terms of privacy, most of the users were happy that their location is tracked for the purpose of improving security with a recommendation of making sure the devices are switched off after working hours. The results showed high potential of the wearables to improve safety and security in cultural events by empowering key personnel such as stewards and police officers with devices connected to the IoT network platform. 


\section{$7 \quad$ Acknowledgement}

The authors would like to thank Veronica Chesi (In-JeT ApS) for her efforts in the development of the impact assessment plan of the MONICA project and her effective engaging of all stakeholders for a constructive user experience management.

\section{$8 \quad$ References}

1. Knecht, K., N, B. and K, S. (2016). A Study on User Experience and Usability of AppleWatch as Wearable Devices.

2. Liang, J., Xian, D., Liu, X., Fu, J., Zhang, X., Tang, B. and Lei, J. (2018). Usability Study of Mainstream Wearable Fitness Devices: Feature Analysis and System Usability Scale Evaluation. JMIR mHealth and uHealth, 6(11), p.e11066.

3. Razvan, G. (2015). Designing A User Experience For Wearable Devices - Usability Geek. [online] Usability Geek. Available at: https://usabilitygeek.com/wearable-devices-userexperience [Accessed 14 Feb. 2019].

4. Schoneveld, A., Frey, N. (2017). IoT Enables Devices and Wearables - MONICA Project. European Commission HORIZON 2020

5. Jahn, M., Michel, O., Suleri, S. (2018). Site Surveys and Pilot Plans for MONICA IoT Platform Pilots - MONICA Project. European Commission HORIZON 2020

6. Leeds Varsity. (2019, August 21). Retrieved from http://LeedsVarsity.com/ 